\title{
Therapy-related mixed phenotype acute leukemia in a pediatric survivor of Ewing sarcoma with a novel RUNX1-TAF3 fusion: A case report and review of the literature
}

Madhav Vissa ${ }^{1}$, Cheryl Peretz ${ }^{1}$, and Jennifer Michlitsch ${ }^{1}$

${ }^{1}$ UCSF Benioff Children's Hospital Oakland

May 8, 2020

\begin{abstract}
Increasing treatment intensity for pediatric Ewing sarcoma (ES) has improved survival, but comes with an increased incidence of secondary malignancy. Here, we describe a case of therapy-related mixed phenotype acute leukemia (t-MPAL), T-myeloid type, in a pediatric patient four years after completion of therapy for ES. Genomic evaluation revealed a novel and likely pathogenic RUNX1-TAF3 fusion. This patient did not respond to T-cell leukemia-directed therapy, and while he initially responded to myeloid leukemia-directed therapy, he never achieved complete remission and died of disease 10 months after diagnosis. Here, we present this case and review prior literature regarding t-MPAL.
\end{abstract}

Full Title: Therapy-related mixed phenotype acute leukemia in a pediatric survivor of Ewing sarcoma with a novel RUNX1-TAF3 fusion: A case report and review of the literature

Authors: Madhav Vissa, MD, Cheryl Cohler Peretz, MD, Jennifer Michlitsch, MD

Author Affiliations: UCSF Benioff Children's Hospital Oakland, Oakland, CA

Corresponding Author:

Jennifer Michlitsch

Address: 747 52nd Street, Oakland, CA 94609

Phone: 510-428-3264

Fax: 510-601-3916

Email: jennifer.michlitsch@ucsf.edu

\section{Word Count:}

Abstract: 98 words

Main Text: 1196 Words

Tables : 2

No figures or supporting information files

Short running title: Pediatric therapy-related MPAL, T-myeloid type

Keywords : therapy-related leukemia, MPAL, AML, secondary malignancy

\section{Abbreviations:}




\begin{tabular}{ll}
\hline ES & Ewing Sarcoma \\
\hline MPAL & Mixed phenotype acute leukemia \\
SMN & Secondary malignant neoplasm \\
AML & Acute myeloid leukemia \\
WHO & World Health Organization \\
MDS & Myelodysplastic syndrome \\
ALL & Acute lymphoblastic leukemia \\
t-MPAL & Therapy-related mixed phenotype acute leukemia \\
MRD & Minimal residual disease \\
COG & Children's Oncology Group \\
CR & Complete Remission \\
HSCT & Hematopoeitic stem cell transplant \\
\hline
\end{tabular}

Title: Therapy-related mixed phenotype acute leukemia in a pediatric survivor of Ewing sarcoma with a novel RUNX1-TAF3 fusion: A case report and review of the literature

Madhav Vissa, MD, Cheryl Cohler Peretz, MD, Jennifer Michlitsch, MD

Abstract:

Increasing treatment intensity for pediatric Ewing sarcoma (ES) has improved survival, but comes with an increased incidence of secondary malignancy. Here, we describe a case of therapy-related mixed phenotype acute leukemia (t-MPAL), T-myeloid type, in a pediatric patient four years after completion of therapy for ES. Genomic evaluation revealed a novel and likely pathogenic RUNX1-TAF3 fusion. This patient did not respond to T-cell leukemia-directed therapy, and while he initially responded to myeloid leukemia-directed therapy, he never achieved complete remission and died of disease 10 months after diagnosis. Here, we present this case and review prior literature regarding t-MPAL.

Introduction:

Modern treatment modalities have improved survival for childhood cancer substantially. In particular, Ewing sarcoma (ES) has seen a dramatic improvement in survival, mostly owing to aggressive local and systemic therapy. ${ }^{1}$ Unfortunately, due to exposure to genotoxic therapy including topoisomerase II inhibitors, alkylating agents, anthracyclines and radiation, treatment for ES carries a significant risk of secondary malignant neoplasms (SMNs), with therapy-related AML/MDS being the most common. ${ }^{2,3}$ Overall survival after diagnosis of secondary leukemia in children ranges from 20-40\%, though some have reported more optimistic outcomes, especially when patients are treated with allogeneic stem cell transplantation. ${ }^{4-11}$ Mixed phenotype acute leukemia (MPAL), defined by the World Health Organization (WHO) as acute leukemia with immunophenotypic expression of both lymphoid ( $\mathrm{T}$ or $\mathrm{B}$ ) and myeloid cell markers, is a rare entity accounting for $3-5 \%$ of de novopediatric acute leukemias. ${ }^{12,13}$ Outcomes of de novo pediatric MPAL are similar to those of de novo AML, though significantly worse than those of ALL. There is controversy over standard MPAL treatment, with many providers favoring ALL-type therapy. ${ }^{12}$ Therapy-related MPAL (tMPAL) is rare and, to our knowledge has been reported only once in a child, who harbored a B-myeloid variant. ${ }^{14}$ Here, we report a case of t-MPAL, T-myeloid type, in a childhood survivor of Ewing sarcoma.

Case Presentation:

A two-year-old male was diagnosed with localized Ewing sarcoma of the left scapula and was enrolled on the Children's Oncology Group (COG) clinical trial AEWS1031. He received systemic vincristine, doxorubicin, cyclophosphamide, etoposide and ifosfamide as well as 5580cGy external beam radiation to the left scapula. His clinical course was complicated by severe veno-occlusive disease/sinusoidal obstruction syndrome, respiratory failure, disseminated varicella and fungal infections, pancreatitis, and cardiomyopathy. He fortunately recovered from these complications and was in complete remission by the end of therapy. 
He was subsequently treated for late effects of therapy including developmental delay, functional limitations with mobility, and transfusion related-iron overload, for which he was briefly phlebotomized.

Four years after completion of therapy for ES, routine labs showed progressive pancytopenia prompting further evaluation. Bone marrow morphology showed hypercellularity with myelofibrosis; flow cytometry showed two abnormal blast populations, both with predominantly T-cell marker expression and minimal myeloid differentiation: cytoplasmic CD3 (majority subset), CD7 (bright), CD33 (uniform), CD34 (variable), CD38, CD45, CD56 (small subset), CCD71, CD117 (variable), CD123 (variable), HLA-DR. There was no significant expression of CD2, surface CD3, CD4, CD5, CD8, CD10, CD14, CD15, CD16, CD19, CD20, CD64, cytoplasmic CD79a, cytoplasmic MPO or surface light chains.

Cytogenetic analysis revealed a clonal population with loss of chromosome 6 , gain of chromosome 13, and a ring chromosome in $60 \%$ of cells. A cancer genetics panel (UCSF500) showed a pathogenic NRASmutation and a novel RUNX1-TAF3 structural variant, predicted to result in a functional fusion gene product and considered likely pathogenic. Germline testing showed no alterations in cancer-related genes. Based on these data, the patient was diagnosed with t-MPAL, T-myeloid type.

A summary of the patient's treatment course is presented in Table 1. Briefly, per current guidelines for de novo pediatric MPAL ${ }^{15}$, he received ALL-type induction therapy without a response. End of induction marrow evaluation showed progression of disease as well as a third clonal population with $17 \%$ of cells expressing monosomy 7. He was re-induced with AML-type therapy and had a significant response, with minimal residual disease (MRD) of $0.09 \%$ by flow cytometry. Subsequent attempts were made to achieve complete remission (CR) with the goal to proceed with allogeneic hematopoietic stem cell transplant (HSCT), however these attempts were unsuccessful. Due to prior cardiotoxicity, he was not a candidate for additional anthracycline therapy. The patient died of disease progression 10 months after diagnosis of t-MPAL at age 8 .

Discussion:

Secondary malignant neoplasms (SMNs) are a rare but potentially devastating complication for survivors of childhood cancer. Therapy-related AML/MDS is the most common SMN and it remains difficult to cure despite use of aggressive therapies, often including HSCT. With Ewing sarcoma in particular, prior studies have shown that survivors of ES have a significantly increased risk of secondary AML. One large cancer registry study reported that the standardized incidence ratio (SIR; observed to expected ratios based on age- and sex-specific incidence in the general population) for AML after ES was 71.17. ${ }^{16}$ Although therapyrelated hematologic malignancies in survivors of childhood cancer have been well described, secondary, or therapy-related MPAL is rare.

We have identified eight prior published cases of t-MPAL, four B-myeloid and four T-myeloid, and summarized major clinical features in Table 2. Cases had variable exposures for treatments of different primary diseases and varied immunophenotypic and cytogenetic characteristics. Most patients initially received AMLdirected therapy. Only two of the nine patients reviewed here (including the present case) were alive at the time of case report: one was diagnosed with a Philadelphia-chromosome positive t-MPAL and was treated with an imatinib-containing regimen; the other underwent HSCT after achieving CR with ALL-type induction therapy. As is the case with de novopediatric MPAL, recurrent chromosomal abnormalities have not been identified.

We present the first published case of t-MPAL of the T-myeloid type in a pediatric patient. This lineage assignment is essential because it helps determine management. Diagnosis of MPAL, however, is evolving and is often not straightforward. In this case, the immunophenotype, with strong expression of cytoplasmic CD3 was highly suggestive of T-cell leukemia. Blasts also expressed myeloid markers, CD33 and CD117, thus it may be argued that these myeloid markers represented aberrant expression rather than defining the lineage. Although our patient did not meet strict WHO 2016 criteria for myeloid leukemia (requires either MPO expression or 2 markers of monocytic differentiation including NSE, CD11c, CD14, CD64 or lysozyme), a number of supporting factors validate the diagnosis of T-myeloid MPAL. The first of these is 
history of treatment for ES, which carries a notable increased risk for AML as mentioned above. Second, this patient responded to myeloid, but not T-lymphoid therapy. Third, a cancer genetics panel (UCSF500) identified an activating mutation in NRAS, which is commonly found inde novo MPAL and AML. Lastly, the leukemia developed five years after alkylator exposure and included a clonal population with monosomy 7. Alkylating agents have been associated with development of AML 5-7 years after exposure, frequently with loss or deletion of chromosomes 5 or $7 .{ }^{17}$

We also identified a novel fusion of RUNX1 with TAF3. RUNX1, on chromosome 21, is a member of the core-binding factor family of transcription factors and is a critical regulator of hematopoiesis. ${ }^{18}$ Mutations in $R U N X 1$ are commonly thought to be drivers of leukemogenesis. Further, structural variants including fusions of RUNX1 are very common in pediatric AML. ${ }^{19}$ Notably, NRAS mutations have been seen in a third of AML patients with certain RUNX1 rearrangements. ${ }^{18}$ TATA-box binding protein associated factor 3, TAF3, on chromosome 10, has been documented in structural variants involving NUP family genes, but not as a fusion partner with RUNX1. This novel fusion places exons 1-7 of $R U N X 1$ in frame with exons 3-7 of $T A F 3$, which is predicted to result in a pathogenic gene product.

This case, the first reported therapy-related T-myeloid MPAL in a pediatric patient, represents the growing importance of molecular diagnostics not only in predicting the behavior of hematologic malignancies, but potentially also in choosing appropriate therapy.

Disclosure of conflicts of interest: None

References

1. Pappo AS, Dirksen U. Rhabdomyosarcoma, Ewing Sarcoma, and Other Round Cell Sarcomas. Journal of Clinical Oncology . Published online December 8, 2017. doi:10.1200/JCO.2017.74.7402

2. Caruso J, Shulman DS, DuBois SG. Second malignancies in patients treated for Ewing sarcoma: A systematic review. Pediatric Blood \& Cancer . 2019;66(11):e27938. doi:10.1002/pbc.27938

3. Bhatia S, Krailo MD, Chen Z, et al. Therapy-related myelodysplasia and acute myeloid leukemia after Ewing sarcoma and primitive neuroectodermal tumor of bone: a report from the Children's Oncology Group. Blood . 2007;109(1):46-51. doi:10.1182/blood-2006-01-023101

4. Kobos R, Steinherz PG, Kernan NA, et al. Allogeneic Hematopoietic Stem Cell Transplantation for Pediatric Patients with Treatment-Related Myelodysplastic Syndrome or Acute Myelogenous Leukemia. Biology of Blood and Marrow Transplantation . 2012;18(3):473-480. doi:10.1016/j.bbmt.2011.11.009

5. Koh K-N, Yoo KH, Im HJ, et al. Characteristics and Outcomes of Second Malignant Neoplasms after Childhood Cancer Treatment: Multi-Center Retrospective Survey. J Korean Med Sci . 2016;31(8):1254. doi:10.3346/jkms.2016.31.8.1254

6. Woodard P, Barfield R, Hale G, et al. Outcome of hematopoietic stem cell transplantation for pediatric patients with therapy-related acute myeloid leukemia or myelodysplastic syndrome. Pediatr Blood Cancer . 2006;47(7):931-935. doi:10.1002/pbc.20596

7. Aguilera DG, Vaklavas C, Tsimberidou AM, Wen S, Medeiros LJ, Corey SJ. Pediatric Therapy-related Myelodysplastic Syndrome/Acute Myeloid Leukemia: The MD Anderson Cancer Center Experience. Journal of Pediatric Hematology/Oncology . 2009;31(11):803-811. doi:10.1097/MPH.0b013e3181ba43dc

8. Tabori U, Revach G, Nathan PC, et al. Toxicity and outcome of children with treatment related acute myeloid leukemia. Pediatr Blood Cancer . 2008;50(1):17-23. doi:10.1002/pbc.21157

9. Cho HW, Choi YB, Yi ES, et al. Therapy-related myeloid neoplasms in children and adolescents. Blood Res . 2016;51(4):242. doi:10.5045/br.2016.51.4.242

10. Hong KT, Choi JY, Hong CR, Kang HJ, Park KD, Shin HY. Therapy-related Acute Myeloid Leukemia After the Treatment of Primary Solid Cancer in Children: A Single-center Experience. J Pediatr Hematol 
Oncol . 2018;40(1):6.

11. Vyas C, Jain S, Kapoor G. Therapy Related AML/MDS Following Treatment for Childhood Cancer: Experience from a Tertiary Care Centre in North India. Indian J Hematol Blood Transfus . 2018;34(1):78-82. doi:10.1007/s12288-017-0840-x

12. Orgel E, Alexander TB, Wood BL, et al. Mixed-phenotype acute leukemia: A cohort and consensus research strategy from the Children's Oncology Group Acute Leukemia of Ambiguous Lineage Task Force.Cancer . Published online October 29, 2019:cncr.32552. doi:10.1002/cncr.32552

13. Arber DA, Orazi A, Hasserjian R, et al. The 2016 revision to the World Health Organization classification of myeloid neoplasms and acute leukemia. Blood . 2016;127(20):2391-2405. doi:10.1182/blood-2016-03643544

14. Whittle SB, Punia JN, López-Terrada D, Gaikwad A, Hampton OA, Heczey A. Therapy-related Acute Leukemia With Mixed Phenotype and Novel t(1: 6)(q25;p23) After Treatment for High-risk Neuroblastoma. J Pediatr Hematol Oncol . 2017;39(8):e486-e488. doi:10.1097/MPH.0000000000000956

15. Hrusak O, de Haas V, Stancikova J, et al. International cooperative study identifies treatment strategy in childhood ambiguous lineage leukemia. Blood . 2018;132(3):264-276. doi:10.1182/blood-2017-12-821363

16. Rihani R, Bazzeh F, Faqih N, Sultan I. Secondary hematopoietic malignancies in survivors of childhood cancer. Cancer . 2010;116(18):4385-4394. doi:10.1002/cncr.25313

17. Barnard D, Woods W. Treatment-related myelodysplastic syndrome/acute myeloid leukemia in survivors of childhood cancer - An update.Leukemia \& Lymphoma . 2005;46(5):651-663. doi:10.1080/10428190500051042

18. Sood R, Kamikubo Y, Liu P. Role of RUNX1 in hematological malignancies. Blood . 2017;129(15):20702082. doi:10.1182/blood-2016-10-687830

19. Tarlock K, Zhong S, He Y, et al. Distinct age-associated molecular profiles in acute myeloid leukemia defined by comprehensive clinical genomic profiling. Oncotarget . 2018;9(41):26417-26430. doi:10.18632/oncotarget.25443

20. Parker C, Waters R, Leighton C, et al. Effect of mitoxantrone on outcome of children with first relapse of acute lymphoblastic leukaemia (ALL R3): an open-label randomised trial. Lancet . 2010;376(9757):2009-2017. doi:10.1016/S0140-6736(10)62002-8

21. Yamamoto K, Sada A, Kawano Y, Katayama Y, Shimoyama M, Matsui T. Therapy-related, mixed phenotype acute leukemia with $\mathrm{t}(1 ; 21)(\mathrm{p} 36 ; \mathrm{q} 22)$ and RUNX1 rearrangement. Cancer Genetics and Cytogenetics . 2010;201(2):122-127. doi:10.1016/j.cancergencyto.2010.05.011

22. Cho J-H, Hur M, Moon H-W, et al. Therapy-related acute leukemia with mixed phenotype and $\mathrm{t}(9 ; 22)$ (q32;q11.2): a case report and review of the literature. Human Pathology . 2012;43(4):605-609. doi:10.1016/j.humpath.2011.07.010

23. Gong X, Yan L, Xiao X, Tang Q, Zhao X. Secondary mixed phenotype acute leukemia following chemotherapy for diffuse large B-cell lymphoma: a case report and review of the literature. :7.

24. Briasoulis E, Tzouvara E, Tsiara S, Vartholomatos G, Tsekeris P, Bourantas K. Biphenotypic Acute Leukemia Following Intensive Adjuvant Chemotherapy for Breast Cancer: Case Report and Review of the Literature. The Breast Journal . 2003;9(3):241-245. doi:10.1046/j.1524-4741.2003.09323.x

25. Colovic N, Suvajdzic N, Kraguljac Kurtovic N, et al. Therapy-related acute leukemia in two patients with multiple sclerosis treated with Mitoxantrone. Biomedicine \& Pharmacotherapy . 2012;66(3):173-174. doi:10.1016/j.biopha.2011.10.004 
26. Roberts III E, Oncale M, Safah H, Schmieg J. Therapy-Related T/Myeloid Mixed Phenotype Acute Leukemia in a Patient Treated with Chemotherapy for Cutaneous Diffuse Large B Cell Lymphoma. JOURNAL OF THE LOUISIANA STATE MEDICAL SOCIETY ::7.

27. Yang D, Cho SR, Jung S, et al. A Case of Therapy-Related Acute Leukemia With Mixed Phenotype With BCR-ABL1 After Treatment of Diffuse Large B-Cell Lymphoma. Ann Lab Med . 2017;37(2):166. doi:10.3343/alm.2017.37.2.166

\section{Hosted file}

Vissa Table 1.docx available at https://authorea.com/users/319637/articles/449341-therapyrelated-mixed-phenotype-acute-leukemia-in-a-pediatric-survivor-of-ewing-sarcoma-with-anovel-runx1-taf3-fusion-a-case-report-and-review-of-the-literature

\section{Hosted file}

Vissa Table 2.docx available at https://authorea.com/users/319637/articles/449341-therapyrelated-mixed-phenotype-acute-leukemia-in-a-pediatric-survivor-of-ewing-sarcoma-with-anovel-runx1-taf3-fusion-a-case-report-and-review-of-the-literature 